\title{
Optimized Analysis of Escort Rout Problem in Commercial Banks
}

\author{
Yulei Yang \\ Urban Vocational College of Sichuan, Chengdu, 610060, China
}

Keywords: Commercial Bank, Escort Rout, Dynamic Network.

\begin{abstract}
China's banking industry continuing to use the thought and mode of planned economy in cash management has led to high operation cost, low management efficiency and huge risk. In order to improve the performance and service of banks, it is helpful to optimize cash distribution activities of commercial banks by modern logistics theory and methods. After building the mathematical model with the randomness of road network and customer satisfaction index, the escort vehicle rout is planned under different traffic intensity and network fluctuation parameters.
\end{abstract}

\section{Introduction}

Before 1997, cash distribution activities of China's financial organizations have been conducted independently by each financial organization until specialized convoy company was established in Shenzhen pilot. Then cash distribution entered into a new era. For twenty years, convoy companies have grown gradually and formed own distinctive features. But there are still huge gaps between Chinese convoy companies and western convoy companies. The biggest difficulty of cash distribution management is empirical planning convoy route. Convoy vehicle's route planning should consider the convoy distribution of n operation branches subordinated to center treasury for the main purpose of securely, rapidly and economically complete convoy work. Random fluctuation of road net and customer satisfaction are important factors influencing convoy service. Thus this paper will abstract the problems to the issue of dynamic network vehicle routing and plan the convoy route of commercial bank's cash distribution actives with dynamic network vehicle routing by combining with the logistics characteristics of cash distribution.

\section{Model building \\ Problem description}

Vehicle running speed can be used to weigh the dynamic change of road network when studying dynamic network vehicle routing issue. The randomness of vehicle running speed is reflected in two forms: one is the fluctuation caused by emergencies and the other one is the routine fluctuation caused by the inherent complexity and unpredictability of road network. In convoy operation, security factor is the most important limitation condition, thus vehicles usually run in high quality roads of urban road network which means the probability of the first situation is very small. It requires greatly increase the time and costs of normal operation of considering this small probability event and it is not cost-effective. So this paper will not take the small probability event into consideration but consider the random fluctuation of running speed caused by the inherent uncertainty of road network.

Through above analysis, this paper set the max lag time allowable for all bank branches as the index of customer satisfaction. With the confirmation of the max lag time value, the upper bound of customer's dissatisfaction is thus confirmed. In practical calculation, handle this problem in following ways. Firstly, add allowable delay limit to the time window of all branches, and calculate the whole road with the min speed in each road section in each time period, and punish those violating the lag time of expanded time window. In this way, the optimal route of allowable delay can be obtained. 


\section{Parameter setting}

Mathematical model established in this paper can be elaborated as follow. In undirected graph $\quad P=(V, R), \quad V=\{0,1, \cdots, n\}$, the aggregate of branches, $R \quad\{(i, j): i, j \in V, i \neq j\}$ is the aggregate of edges. Branch 0 refers to the location of center treasury, where $\mathrm{m}$ completely same convoy vehicles with loading capacity of Q; branch $\mathrm{i}(\mathrm{i} \in \mathrm{V}$ has non-negative quantity demanded $\mathrm{q}_{\mathrm{i}}\left(\mathrm{q}_{\mathrm{i}} \leqslant \mathrm{Q}\right)$, and the allowable serving time window is $\left\{\mathrm{a}_{\mathrm{i}}, \mathrm{b}_{\mathrm{i}}\right\}$, required service time is $\mathrm{t}_{\mathrm{i}}$; Distance matrix $\quad d_{i j}$ is defined in the edge set $R$, and $d_{i j}=d_{j i}$; the whole convoy time is divided into $\mathrm{p}$ sections, denoted as $\quad\left\{\mathrm{T}_{1}, \mathrm{~T}_{2}, \cdots \mathrm{T}_{\mathrm{p}}\right\}$; running speed matrix $\quad \mathrm{V}_{\mathrm{ij}} \mathrm{T}$ is defined by edge set $\mathrm{R}$ and the set of time period $\left\{\mathrm{T}_{1}, \mathrm{~T}_{2}, \cdots \mathrm{T}_{\mathrm{p}}\right\}$; then divide $\mathrm{R}$ into $\mathrm{C}$ subsets $\quad \mathrm{R}_{\mathrm{c}}(1 \leqslant \mathrm{c} \leqslant \mathrm{C}$, i.e. if road section $(i, j) \in R_{c}$, then within the time period $T$, running speed in $(i, j)$ is $v_{i j T}=v_{c T}$; actual running speed is shown in graph 1 . It is a random variable which is processed as a discrete random variable in this paper. The state space of $\quad\left\{\mathrm{v}_{\mathrm{cT} 1}, \mathrm{v}_{\mathrm{cT} 2}, \ldots, \mathrm{v}_{\mathrm{cTk}}\right\}$ contains k elements.

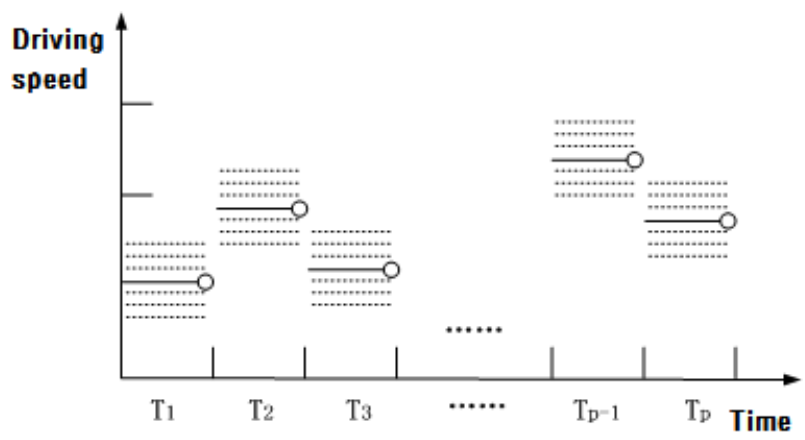

Fig. 1. Schematic diagram of model running speed

\section{Mathematical model}

Set up following mathematical model based on above hypothesis and parameter setting.

Objective function:

$$
\min \mathrm{P}=\sum_{\mathrm{i}=1}^{\mathrm{n}} \sum_{\mathrm{i}=1}^{\mathrm{n}} \sum_{\mathrm{k}=1}^{\mathrm{m}} \overline{\mathrm{f}_{\mathrm{ij}}}\left(\overline{\mathrm{s}_{\mathrm{i}}}+\mathrm{t}_{\mathrm{i}}\right) \mathrm{z}_{\mathrm{ijk}}+\mathrm{c}_{\mathrm{f}} \sum_{\mathrm{k}=1}^{\mathrm{m}} \mathrm{y}_{\mathrm{k}}
$$

Constraint condition:

$$
\begin{array}{cc}
\sum_{\mathrm{i} \neq \mathrm{i}}^{\mathrm{n}} \mathrm{z}_{\mathrm{ijk}}=\sum_{\mathrm{i} \neq \mathrm{i}}^{\mathrm{n}} \mathrm{z}_{\mathrm{jik}} & (\forall \mathrm{k}=1,2, \cdots, \mathrm{m}, \quad \forall \mathrm{i}, \mathrm{j} \in \mathrm{V} \backslash\{0\}) \\
\sum_{\mathrm{k}=1}^{\mathrm{m}} \sum_{\mathrm{i} \neq \mathrm{j}}^{\mathrm{n}} \mathrm{z}_{\mathrm{ijk}}=1 & (\forall \mathrm{k}=1,2, \cdots, \mathrm{m}, \quad \forall \mathrm{i}, \mathrm{j} \in \mathrm{V} \backslash\{0\}) \\
\sum_{\mathrm{i}}^{\mathrm{n}} \mathrm{z}_{0 \mathrm{ik}}=1 & (\forall \mathrm{k}=1,2, \cdots, \mathrm{m}, \quad \forall \mathrm{i}, \mathrm{j} \in \mathrm{V} \backslash\{0\}) \\
\sum_{\mathrm{i}}^{\mathrm{n}} \mathrm{z}_{\mathrm{i} 0 \mathrm{k}}=1 & (\forall \mathrm{k}=1,2, \cdots, \mathrm{m}, \quad \forall \mathrm{i}, \mathrm{j} \in \mathrm{V} \backslash\{0\}) \\
\sum_{\mathrm{i} \neq \mathrm{h}}^{\mathrm{n}} \mathrm{z}_{\mathrm{ihk}}=\sum_{\mathrm{i} \neq \mathrm{h}}^{\mathrm{n}} \mathrm{z}_{\mathrm{jhk}} & (\forall \mathrm{k}=1,2, \cdots, \mathrm{m}, \quad \forall \mathrm{i}, \mathrm{j}, \mathrm{h} \in \mathrm{V} \backslash\{0\}) \\
\widetilde{\mathrm{s}_{\mathrm{i}}}+\mathrm{t}_{\mathrm{i}}+\widetilde{\mathrm{f}_{\mathrm{ij}}}\left(\widetilde{\mathrm{s}_{\mathrm{i}}}+\mathrm{t}_{\mathrm{i}}\right)-\mathrm{M}\left(1-\sum_{\mathrm{k}=1}^{\mathrm{m}} \mathrm{z}_{\mathrm{ijk}}\right) \leqslant \widetilde{\mathrm{s}_{\mathrm{i}}}(\forall \mathrm{k}=1,2, \cdots, \mathrm{m}, \quad \forall \mathrm{i}, \mathrm{j} \in \mathrm{V} \backslash\{0\}) \\
\mathrm{a}_{\mathrm{i}} \leqslant \widetilde{\mathrm{s}_{\mathrm{i}}} \leqslant \mathrm{b}_{\mathrm{i}}+\mathrm{L} & (\forall \mathrm{i} \in \mathrm{V} \backslash\{0\}) \\
\sum_{\mathrm{k}=1}^{\mathrm{m}} \mathrm{y}_{\mathrm{k}} \leqslant \mathrm{m} & (\forall \mathrm{k}=1,2, \cdots, \mathrm{m})
\end{array}
$$

Objective function (1) is expectation running time plus the vehicle fixed charge, "-"used to represent the expectation value. $\overline{\mathrm{s}_{\mathrm{i}}}$ represents the average time of convoy vehicles arriving at branches in long-term operation. Constraint condition (2) ensures in-out balance in each branch, i.e. the number of convoy vehicles arriving at branches equals to the number leaving branches. Constraint 
condition (3) shows that each vehicle can only visit one branch. Constraint condition (4) and (5) show that all convoy vehicles start off in the position of centre treasury and get back after finishing distribution. Constraint condition (27) shows that the time of starting service cannot be earlier than the lower limit of branch time window, i.e. vehicles arriving in advance must wait, " $"$ used to represent the value in the worst situation. Constraint condition (8) shows that time window limit is expanded in the worst situation. Constraint condition (9) shows that the total number of convoy vehicles cannot exceed the sum of vehicles parking in centre treasury [26].

As objective function involves the calculation of expectation value, it will be very hard to obtain the exact value. Thus this paper will conduct numerical calculation by means of Monte Carlo simulation. As long as simulation times are enough, we can obtain the expectation time value in the given route. Next, use genetic algorithm to solve the whole model.

\section{Empirical research}

Through field investigation and data collection in a commercial bank of a city, verify and analyze the mathematical model established in this paper.

\section{Information of operation branch}

There are totally 30 operation branches of the commercial bank of a city, including 7 county-level branch and 1 non-local branch. The centre treasury of the city conducts convoy and distribution to the rest 22 bank operation branches due to regional limitation. In addition, the centre treasury also serves 3 large-scale enterprises. Through investigation on the convoy condition of the commercial bank of the city in May 2016, this paper has summarized the trunk convoy condition of 25 branches serviced by bank's centre treasury in May. When calculating, randomly select a number from all corresponding trunk number of each branch probably each time as the demanded quantity of the branch.

\section{Service time window}

As convoy service is different with ordinary logistics distribution activities, the time of convoy should be strictly controlled to ensure the normal operation of each branch. Through investigation, each branch of the commercial bank has specific time for receiving trunk. Meanwhile, to share the stress of early preparing for receiving trunk, the convoy route will be changed every three months, which can improve the confidentiality of convoy routing and also can help each branch be the first to receive trunk.

Meanwhile, due to strict procedures for trunk handover, staffs of both sides must be very familiar with the operation procedures. So the service time is relevantly fixed. According to statistics, the time for handing over trunk is about 1 min 30 seconds. Another 30 seconds is the time consumed during the convoy vehicle's speedup or slow-down. This paper added up the exampled service time as 3mins. Assume the time of vehicle arriving at customer I isti, then set its time window as[ti-1, ti+1]. Then it can satisfy that each customer's time window width is 2 mins averagely.

\section{Time-dependent running speed}

Assume $\quad \overline{V_{\min }}$ is the expectation speed in peak hours, $\quad \overline{\mathrm{V}_{\max }}$ is the expectation speed in nonpeak hours, then the average speed in the road $\quad V_{\text {ave }}=\frac{1}{3} \overline{V_{\min }}+\frac{2}{3} \overline{V_{\max }}$, but $\frac{\overline{V_{\max }}}{V_{\min }}$ is the ratios between the speed in peak hours and the speed in nonpeak hours. It can reflect traffic loading rate, i.e. the intensity of time dependency. Actually the whole time period of convoy vehicle's running can be regarded as peak time. The peak and nonpeak here are relative. The actual running speed in each time period will fluctuate near the expectation speed $\frac{\overline{V_{\max }}}{V_{\min }}$. The relative deviation of fluctuation is determined by parameter $a$. The larger value of $a$, the greater uncertainty in this road section. 
Due to no access to detailed road network information, this paper has divided the road set based on the length of road section and estimate the running speed in different kinds of roads based on the

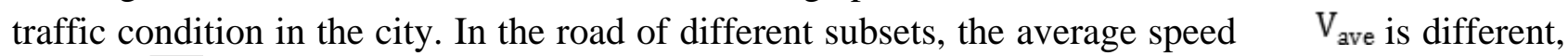
and $\quad \frac{\overline{V_{\max }}}{V_{\min }}$ is also different. The last three lines list the ratio between the intensive max speed and min speed in each road under high, medium and low traffic loading. It is the fastest in main roads during nonpeak time. But as peak time approaches, there are more vehicles and then the speed has decreased most. On the contrary, it is the slowest in branch roads but few vehicles are added in peak time, so the speed decrease is not obvious.

\section{Calculation result}

During model solution, add the convoy operation costs to the punishment caused by lag time to reflect the quality of solution. Set the fixed cost of each vehicle is 1000 , fine for unit lag time is 500 , and the cost in unit running time is 5 . This paper did not use the coefficient ratio in ordinary VRP model to guarantee the cost minimized as the first optimal object. Instead, the paper has set the fixed vehicle costs as relevantly small umber to guarantee the first optimal object that running time of convoy vehicle is the minimum. Set the evolving total algebra as 100, the continuous non-modified algebra is 10. Calculation results are shown in table 1.

Table 1. Calculation results

\begin{tabular}{|c|c|c|c|c|c|}
\hline & $\alpha$ & $\begin{array}{c}\text { Number of } \\
\text { vehicles }\end{array}$ & Running time & Max lag time & Total cost \\
\hline \multirow{2}{*}{$\begin{array}{c}\text { Low traffic } \\
\text { loading }\end{array}$} & $20 \%$ & 3 & 91.26 & 11.17 & 9041.3 \\
\cline { 2 - 6 } & $25 \%$ & 3 & 98.93 & 11.63 & 9309.65 \\
\hline \multirow{2}{*}{$\begin{array}{c}\text { Medium } \\
\text { traffic } \\
\text { loading }\end{array}$} & $30 \%$ & 3 & 106.54 & 12.32 & 9692.7 \\
\cline { 2 - 6 } & $20 \%$ & 3 & 117.75 & 12.76 & 9968.75 \\
\hline \multirow{2}{*}{$\begin{array}{c}\text { High traffic } \\
\text { loading }\end{array}$} & $25 \%$ & 4 & 125.62 & 13.18 & 11218.1 \\
\cline { 2 - 6 } & $20 \%$ & 4 & 136.01 & 13.69 & 11525.05 \\
\hline \multirow{2}{*}{} & $30 \%$ & 4 & 152.38 & 14.05 & 13167.9 \\
\hline
\end{tabular}

Before optimization, banks use empirical planning method. The average running time is 115 mins under low traffic loading, 140mins under medium traffic loading, and 180 mins under high traffic loading. It can be seen that the convoy routing planned in this paper is better than original routing under low and medium traffic loading. But under high traffic loading, the convoy routing in this paper has no obvious advantages. Thus it is suggested to combine two methods to plan convoy routing in practical operation.

\section{Conclusion}

Dynamic network vehicle routing model can solve the time dependency and randomness of road network, help convoy vehicles to make routing planning. In addition, the larger allowable limit of delay, the greater of the freedom degree of route planning. In this way, it can reduce the expected self-running costs but may reduce service level. Dynamic network vehicle routing model can provide effective decision-making support for balancing the relationship between both. In front of different traffic loading degrees and different uncertain degree of road network, the number of convoy vehicles, expected running time and total cost will be different. Dynamic network vehicle routing model can make correspondingly routing planning according to different conditions. 


\section{References}

[1] Ren Wenchao. VPOWER creates new model of cash logistics, China Logistics and Purchasing, 2005(9).

[2] Zou Weihua. Optimization of number of bank convoy vehicles based on genetic algorithm, Jilin University, 2006.

[3] Wang Long. Primary discussion on Chengdu municipal cash logistics management, Southwestern University of Finance and Economics. 2004.

[4] Wu Shuping. Studies on the financial convoy vehicle scheduling, Tianjin University Of Finance \& Economics, 2005.

[5] Zhang Hong. Growing giant-- hidden dangers and development concept of Chinese financial convoy industry, China Security Service, 2006(13).

[6] Kong Jun. Discussion on Chinese and foreign security convoy enterprises' strategies in China, University of International Business and Economics, 2004.

[7] Liu Wei, Xiong Yanhua. Studies on site selection of logistics center and vehicle scheduling synthesis problems based on genetic algorithm, MIE of China, 2008, 37(19).

[8] Chung-Lun Li, King-Wah Pang, Zhou Xu. Ship Routing Problem with Berthing Time Clash Avoidance Constraints, International Journal of Production Economics, 2011,131(2).

[9] Feng Quanxi, Liu Cheng. Studies on parallel genetic algorithm of logistics distribution vehicle routing problems, Journal of Railway Science and Engineering. 2005, 2(4).

[10]Jiang Dali, Yang Xilong, Du Wen. Studies on genetic algorithm of vehicle routing problems, System Engineering Theory and Practice,1999, 19(2). 